\title{
Admission Control and Concurrent Scheduling for IPTV over mmWave-based WPANs
}

\author{
Lin X. Cai ${ }^{1}$, Lin Cai ${ }^{2}$, Xuemin (Sherman) Shen ${ }^{1}$ and Jon W. Mark ${ }^{1}$ \\ Centre for Wireless Communications, Dept. of Electrical \& Computer Engineering ${ }^{1}$, \\ University of Waterloo, Waterloo, ON N2L 3G1, Canada \\ \{lcai, xshen, jwmark\}@ bbcr.uwaterloo.ca \\ Dept. of Electrical \& Computer Engineering ${ }^{2}$, \\ University of Victoria, Victoria, BC V8W 3P6, Canada \\ cai@ece.uvic.ca*
}

\begin{abstract}
Communications at $60 \mathrm{GHz}$ millimeter-wave (mmWave) band is a promising technology for future wireless personal area networks (WPAN) supporting high data rate applications. Internet Protocol TV (IPTV) is anticipated to be one of the next killer applications, which requires high data rate and stringent quality of services $(Q o S)$ in terms of delay and loss. In this paper, we propose how to efficiently support IPTV flows in a mmWave-based WPAN with QoS guarantee, considering the characteristics of both the IPTV traffic and the mmWave communication technology. We first quantify the effective bandwidth of IPTV video sources using a simple, two-level Markov traffic model. By considering the overheads of the protocol stack in mmWave WPANs, we then quantify the minimal channel time needed for each IPTV flow. Since mmWave-based WPANs will deploy directional antennae to not only extend the transmission range, but also achieve higher spatial multiplexing gain, we further propose an admission control scheme and scheduling algorithm which take advantage of concurrent transmissions. Extensive simulations with NS-2 using real video traces have validated our analysis and demonstrate the efficiency and effectiveness of the proposed schemes, which will be an enabling technology for future mmWave-based WPANs supporting IPTV services.
\end{abstract}

\footnotetext{
*Permission to make digital or hard copies of all or part of this work for personal or classroom use is granted without fee provided that copies are not made or distributed for profit or commercial advantage and that copies bear this notice and the full citation on the first page. To copy otherwise, to republish, to post on servers or to redistribute to lists, requires prior specific permission and/or a fee. Qshine08 July 28-31, 2008, Hong Kong, China. Copyright 2008 ICST ISBN 978-963-9799-26-4 DOI 10.4108/ICST.QSHINE2008.3935
}

\section{Introduction}

Communications using the $60 \mathrm{GHz}$ band is called millimeter-wave (mmWave) communications. FCC recently approved the license-free usage of the spectrum of $57-63 \mathrm{GHz}$, which opens up numerous opportunities for future high data rate (up to a few Gigabit), short-distance, wireless services. Because of the strong interests of using the mmWave technologies for future last-meter wireless access, the IEEE 802.15.3 Task Group 3c (TG3c) was formed in March 2005 to develop a mmWave-based Wireless Personal Area Network (WPAN) Standard, which further promotes the mmWave technologies [1]. On the other hand, one of the future killer applications is the Internet Protocol TV (IPTV): high-definition (HD) TV programs and video streams will be transfered over the ubiquitous IP networks, anywhere, anytime. IPTV applications have very high data rate and stringent Quality of Services (QoS) requirements, which may not be possible to deliver in traditional, low data rate wireless systems. Therefore, the mmWave-based WPAN is considered an ideal candidate for last-meter IPTV distribution, because of its high capacity, high spatial reuse capability, and low interference with other wireless systems and electronic devices.

The ever-increasing popularity of wireless multimedia services leads to the ever-growing density of wireless devices. For instance, in broadband hotspots such as airport and soccer stadium, users can view (on demand) a variety of video media and exchange rich multimedia information with their neighbors. Thus, researchers are striving to push up the limit of the number of users/applications that can share the available wireless resources. In other words, given the mmWave communication channels, it is of critical importance to design network protocols to efficiently utilize the resources and ensure the user-perceived 
QoS of multimedia applications. In [2], an exclusive region based resource allocation scheme is proposed to exploit the spatial multiplexing gain of mmWave WPANs supporting persistent traffic flows. Although video transmission over wireless channel has been extensively studied in the literature [3], efficient admission control and scheduling schemes for high quality video over mmWave-based WPAN is still an open area, which is the major motivation of this work.

The main contributions of this paper are as follows. First, we investigate the traffic characteristics and QoS requirements of IPTV, and the special features of mmWave-based WPAN. Then, we quantify the minimal channel time that should be allocated to each IPTV flow to ensure its QoS. Second, a novel scheduling algorithm allowing concurrent transmissions is proposed, which exploit the spatial multiplexing gain of mmWave-based WPANs using directional antennae. In addition, extensive simulations with NS-2 simulators using real HD video traces have demonstrated the effectiveness and efficiency of the proposed resource allocation and scheduling schemes.

The remainder of the paper is organized as follows. In Section 2, we present an overview of the network architecture and unique features of mmWave-based WPANs; we further investigate the IPTV traffic characteristics and describe a simple traffic model for IPTV. In Section 3, we first analyze the effective bandwidth for an IPTV flow, i.e., the minimal bandwidth that can guarantee its QoS requirements. Then, we calculate the minimal channel time that should be allocated to each flow, considering its effective bandwidth and system overheads. Next, we propose a scheduling algorithm that allows concurrent transmissions efficiently. Simulation results with NS-2 are presented in Section 4 , followed by concluding remarks and future work in Section 5.

\section{System Model}

\section{1 mmWave-based WPANs}

The IEEE 802.15.3 standard defines the basic structure and MAC protocol of WPANs [4]. In this subsection, we first present an overview of the standard and then discuss the special features of mmWave-based WPANs.

A hybrid MAC protocol is adopted in the IEEE 802.15.3 WPAN standard: it uses random access periods for network initiation/association and resource requests, etc., and contention-free periods for scheduled data transmission. Several wireless devices can autonomously form a piconet in which one of them is selected as the piconet coordinator (PNC). The PNC can collect global information about the piconet and allocate wireless resources and schedule transmissions for all devices in the piconet according to their requirements. At the scheduled channel time, devices can communicate in a peer-to-peer fashion. Such a semi-ad hoc

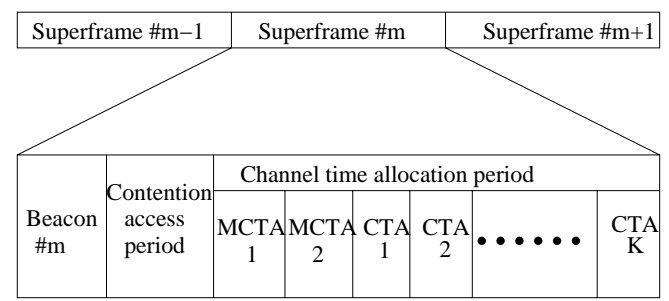

MCTA: Management Channel Time Allocations CTA: Channel Time Allocations

\section{Figure 1. IEEE 802.15.3 Superframe Structure}

setting can provide better QoS than a pure ad hoc network.

Timing in the 802.15.3 WPAN is based on the superframe structure, which is illustrated in Fig. 1. IEEE 802.15.3 defines two methods for communicating data between devices [4]: a) sending asynchronous data or communicating commands in the contention access period (CAP), if present; b) allocating channel time for isochronous and asynchronous streams in the channel time allocations period (CTAP). Although both commands and asynchronous data can be transmitted in the CAP, it is recommended that only commands be transmitted to minimize the length of contention period. This is desirable for reducing the protocol overheads and potential collisions. In addition, for multimedia applications like IPTV, devices may need channel times on a regular basis, and they send channel time requests during the CAP to reserve isochronous channel time in the CTAP. Based on the successfully received requests from all devices, the PNC will schedule and allocate channel time in the CTAP to all devices in a Time Division Multiple Access (TDMA) manner.

For mmWave-based WPANs, we should further consider their special features and design resource management schemes and network protocols accordingly. First, since Oxygen absorption peaks at around $60 \mathrm{GHz}, \mathrm{mmWave}$ fades much faster in the air than lower frequency signals. Thus, directional antenna and associated techniques are not only useful, but essential for mmWave to extend transmission range and improve transmission quality. Second, in high dense mmWave WPANs, multi-hop relay is preferable and necessary to combat high path loss and shadowing effects, so transmissions can occur in a peer-to-peer fashion. With directional antennae, we can further exploit spatial multiplexing gain and allow the peer-to-peer transmissions occur concurrently. Therefore, in our proposed scheduling algorithm, we will consider the usage of directional antenna to schedule confliction-free concurrent transmissions that can significantly improve the resource utilization efficiency and guarantee the QoS requirements of IPTV. 


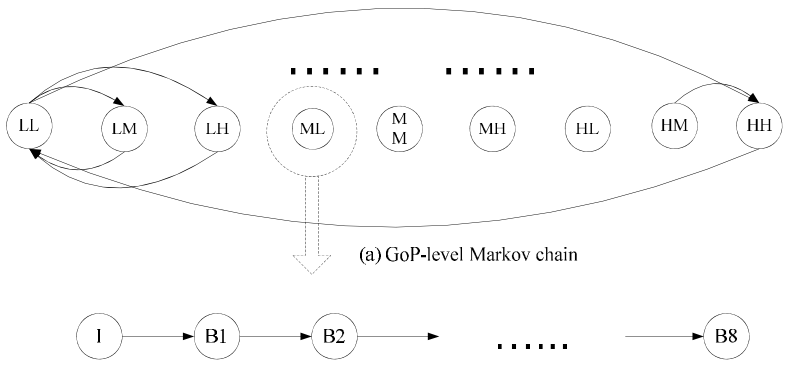

(b) Frame-level Markov chain

Figure 2. Two-level Markov Model for IPTV Traffic

\section{$2.2 \quad$ IPTV traffic model}

IPTV traffic with HD content is typically encoded by MPEG-4 (H.264), which has a high compression ratio, resulting in highly variable data rates (VBR) for the compressed videos. A continuous video stream is sampled into a sequence of frames as the input of the encoder. The main task of a video coder is to remove the spatial and temporal redundancy within each and consecutive frames to save bandwidth. After encoding, frames are emitted periodically comprising Group Of Pictures (GoP). Each GoP contains an I frame and a number of $\mathrm{P}$ and $\mathrm{B}$ frames. For example, with an MPEG codec, the generic GoP is $I B_{1} B_{2} P_{1} B_{3} B_{4} P_{2} B_{5} B_{6} P_{3} B_{7} B_{8}$. The first frame in each GoP is an I frame, which is intra-coded without reference to any other frames. The $\mathrm{P}$ frames are both intra-coded and inter-coded with respect to the previous P or I frame. The $\mathrm{B}$ frames are also intra-coded and inter-coded, and they use both the previous and following P or I frames as references. Since only I frames are encoded without exploiting temporal redundancy, typically an I frame has the largest frame size in each GoP.

A good IPTV traffic model should be able to consider the temporal and spatial correlations of video, and the interGoP and intra-GoP correlations. In [5], a simple, two-level Markov model was proposed for IPTV traffic, which can be used to derive the effective bandwidth of an IPTV flow and can be easily incorporated to any network simulators. Here, we briefly describe the steps to obtain the traffic model which contains a GoP-level Markov chain and a frame-level Markov chain.

In the GoP level, video data rate depends on the texture and motion complexity of the video content, or its spatial and temporal domain correlations. In the spatial and temporal domains, we categorize the video into a number of levels, $S$ and $T$, respectively. Thus, we can use $S \times T$ states to represent the correlations in both domains. Since the duration of a GoP is less than half a second, the spatial and temporal correlations of the video source in each
GoP are assumed to be at the same level, or in the same state. Experimental results show that choosing $S=3$ and $T=3$ makes a good trade-off between model accuracy and complexity. The three correlation levels in each domain are denoted as low $(L)$, medium $(M)$ and high $(H)$ states, as shown in Fig. 2. In the spatial domain, since only the I frames are independently intra-coded, the I frame size is used to determine the texture complexity of the entire GoP. In the temporal domain, the ratio of size of the first $\mathrm{P}$ frame, $P_{1}$, to the I frame size in the same GoP is used to indicate the temporal correlation. This is because $P_{1}$ and I have similar texture complexity, and the ratio of their frame sizes reflect the motion vector from the I frame to the following $P_{1}$ frame.

To capture the intra-GoP correlation, a frame-level Markov chain is established. The time step for the framelevel Markov chain equals the duration of a video frame. Each state in the GoP-level model corresponds to a 12-step Markov chain at the frame-level. The size of an I frame is determined by the spatial domain correlation only. Therefore, the I frame sizes in states $X L, X M$, and $X H$ are the same for $X=L, M, H$ in the spatial domain. A simple method to determine the frame size of each I frame state is to average the size of all I frames belonging to that state. Similarly, we can determine the $P_{1}$ frame size for each state using averages. For the remaining $\mathrm{B}$ and $\mathrm{P}$ frames, since the correlation coefficients of frames in the same GoP are very high, the remaining $\mathrm{P} / \mathrm{B}$ frame sizes in each state are generated based on the $P_{1}$ frame size using the linear equations:

$$
\overline{F_{T}^{K}}=\alpha_{T}^{K} \overline{P_{1}^{K}}, \text { for } T \in\left\{B_{1}, B_{2}, \ldots, B_{8}, P_{2}, P_{3}\right\},
$$

where $\alpha_{T}^{K}$ are constant coefficients determined by the average frame size of $T$.

The discrete-time Markov model can be translated to a continuous-time one, and the transition rate matrix $M$ of the continuous time Markov chain is given by

$$
\mathbf{M}=f(\mathbf{P}-\mathbf{I})
$$

where $f$ is the frame frequency of the video source, and $\mathbf{I}$ is the identity matrix.

\section{Resource Allocation and Scheduling}

\subsection{Effective bandwidth for IPTV source}

Since IPTV traffic is very bursty and IPTV applications have very stringent QoS requirements, appropriate resource allocation is necessary. Effective bandwidth of an IPTV flow is the minimal bandwidth that can ensure its QoS requirements.

First, since IPTV packets suffering excessive delay are useless and have to be discarded by the receiver, we should 
determine the maximal queueing delay in the bottleneck link, and calculate the maximal buffer size that can bound the queueing delay. Second, with high efficient video coding, high packet loss rate will severely degrade the userperceived video quality. In [6], the industry standard requires that the packet loss rate (PLR) of IPTV should be below $10^{-6}$. Therefore, we need to quantify the buffer overflow probability to limit the PLR.

Given the two-level Markov model for IPTV traffic, we can quantify the buffer overflow probability using the fluidflow approach [7].

Let $F_{i}(x)$ denote the probability that the queue length is less than $x$, given that the video source is in state $i(\in\{1,2, \cdots, N\}) . \quad \mathbf{F}(x)$ is the row vector $\left[F_{0}(x) F_{1}(x) \ldots F_{N}(x)\right]$. As shown in [7],

$$
\frac{d \mathbf{F}(x)}{d x} \mathbf{D}=\mathbf{F}(x) \mathbf{M}
$$

where $\mathbf{D}$ is an $N \times N$ diagonal matrix

$$
\mathbf{D}=\operatorname{diag}\left\{\mathrm{D}_{1}-\mathrm{C}, \mathrm{D}_{2}-\mathrm{C}, \ldots, \mathrm{D}_{\mathrm{N}}-\mathrm{C}\right\}
$$

$N=108$ using the two-level Markov model. $C$ is the allocated bandwidth and $D_{i}$ is the source rate generated in state $i$, which equals the frame size of the state over the frame duration. Thus, the buffer overflow probability is given by [7]

$$
\begin{aligned}
G(x) & =1-F(x) \\
& =-\sum_{i: \operatorname{Re}\left[z_{i}<0\right]} a_{i} \sum_{j=1}^{M} \phi_{i j} \exp \left(z_{i} x\right),
\end{aligned}
$$

where $z_{i}$ and $\vec{\Phi}_{i}$ are the eigenvalue and eigenvector of $\mathrm{MD}^{-1}$, respectively, and $a_{i}$ 's are the coefficients that can be derived according to the conditions that $F_{i}(0)=0$ for the overload states.

Since the PLR is a non-increasing function of $C$, given the buffer size and the traffic model, we can use a simple binary search algorithm to determine the minimal $C$ (i.e., the effective bandwidth of the flow) that satisfies the delay and PLR requirements.

\subsection{Resource reservation in mmWave WPANs}

Given the effective bandwidth calculated in the above subsection, we need to quantify the number of time slots reserved for each flow, considering the achievable raw data rate of the communication channel and the overheads from the protocol stack. The result should also reveal the impact of system parameters on resource utilization, which will be useful guidelines for network planner and service providers.

First, with large bandwidth, the PHY layer of mmWavebased WPANs will use different spreading factors and Forward Error Correction (FEC) codes to adapt the raw data

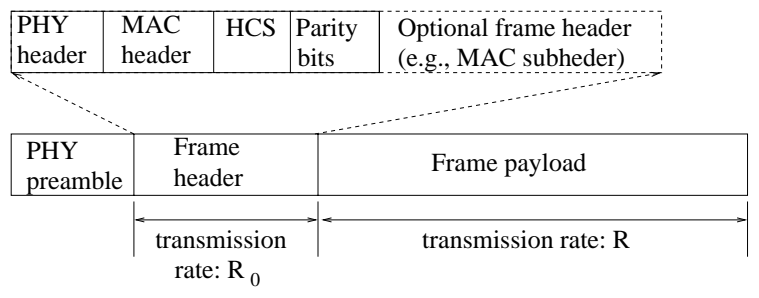

Figure 3. Frame Structure

rate according to the received signal-to-noise ratio (SNR). For instance, in [8], using the single carrier mode, the mandatory data rate can be adapted in the range of $50 \mathrm{Mbps}$ to 1.5 Gbps. According to the Shannon capacity of Additive White Gaussian Noise (AWGN) channel, we obtain the achievable raw data rate $R$ :

$$
R=\eta W \log _{2}(S N R+1),
$$

where $W$ is the signal bandwidth, and $\eta(<1)$ indicates the efficiency of the transceiver design.

The average received SNR can be estimated using the path-loss model:

$$
S N R=\frac{P_{t} G_{t} G_{r} \cdot d^{-\alpha}}{P_{n}}
$$

where $P_{t}$ is the transmission power, $G_{t}$ and $G_{r}$ are the transmitter and receiver antenna gains, respectively, $d$ is the transceiver distance, $\alpha$ is the path loss exponent, and $P_{n}$ is the noise power. For directional antenna, the antenna gain is proportional to $2 \pi / \theta$, where $\theta$ is the radiation angle of the antenna.

Next, we should consider the PHY and MAC overheads in a mmWave WPAN to determine the number of time slots reserved for an IPTV flow, according to its effective bandwidth. As shown in Fig. 3, for each link-layer frame, the overhead includes preamble transmission time, frame headers (which include PHY header, MAC header, HCS, parity symbols, and optional frame header), the short inter-frame space (SIFS), and acknowledgement (ACK). Since frame header is typically transmitted using the basic data rate $R_{0}$ (e.g., $28 \mathrm{Mbps}$ ) which may be much lower than the raw data rate (up to a few Gbps), it is desirable to aggregate a number of data packets into a single frame to reduce the overhead.

Let $n$ be the maximal number of packets (subframes) that can be aggregated in a frame and $P$ be the size of each subframe. If the superframe duration is $S_{T}$, the minimal time slots $(s)$ reserved for each flow with effective bandwidth $C$ should be

$s=\left\lceil\frac{C \cdot S_{T}}{n P}\left(\frac{n P}{R}+\frac{\text { frame header }}{R_{0}}+\mathrm{SIFS}+\mathrm{ACK}\right)\right\rceil$ (slots). 


\subsection{Admission control and concurrent scheduling algorithm}

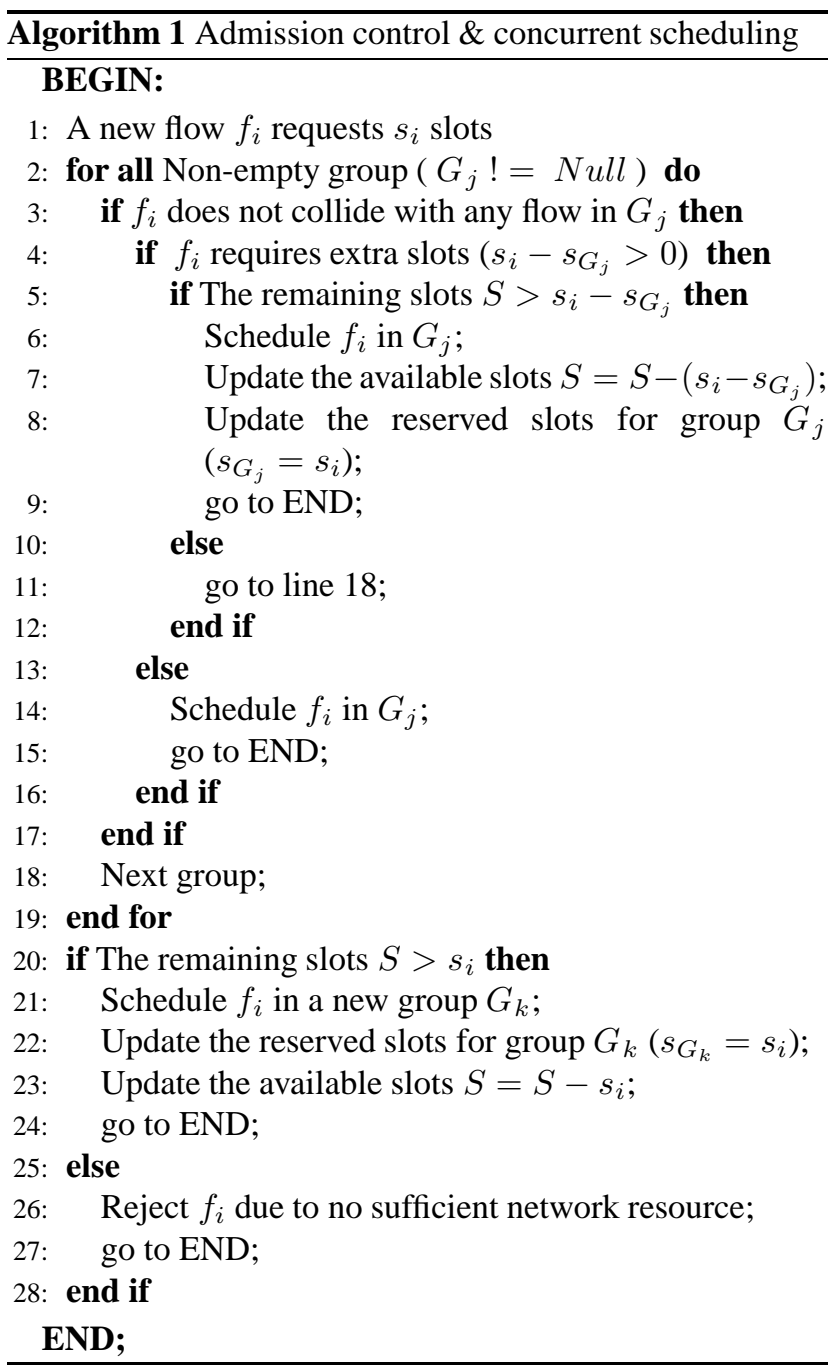

Given the required channel time for each flow, the PNC can use admission control and appropriate scheduling schemes to ensure the QoS of all admitted flows. Two flows can concurrently transmit if the data and ACK transmitted of one flow do not interfere with those of the other flow. With directional antenna, we can assume two flows are conflict-free if the radiation angles of any two nodes belonging to two flows do not point to each other. Therefore, in mmWave-based WPAN with directional antenna, we should exploit the spatial multiplexing gain by efficiently scheduling concurrent transmissions. The pseudo-random code for the admission control and concurrent scheduling algorithm is given in Algorithm 1.

Initially, the total available slots per superframe is $S$. When the first flow $f_{0}$ requests $s_{0}$ slots, the scheduler (PNC) checks if there is enough available network resources to
Table 1. Simulation Parameters

\begin{tabular}{|c|c||c|c||}
\hline$W$ & $500 \mathrm{MHz}$ & $d_{\text {ref }}$ & $1.5 \mathrm{~m}$ \\
\hline$P_{t}$ & $0.1 \mathrm{~mW}$ & $\eta$ & 0.293 \\
\hline$N$ & $-117 \mathrm{dBm} / \mathrm{MHz}$ & $\theta$ & 90 degrees \\
\hline$\alpha$ & 2 & $S$ & 1000 slots \\
\hline$P L_{0}$ & $71.5 \mathrm{~dB}$ & $S_{T}$ & $20 \mathrm{~ms}$ \\
\hline
\end{tabular}

support this flow and schedules it in the first group $G_{0}$ if $S>s_{0}$. The scheduler allocates $s_{0}$ slots to flows in $G_{0}$, $s_{G_{0}}=s_{0}$, and updates the available number of slots by $S \leftarrow S-s_{0}$. Without loss of generality, for any new flow, $f_{i}$, requesting $s_{i}$ slots, the scheduler checks if $f_{i}$ satisfies the concurrent transmission condition with the existing flows. If $f_{i}$ can concurrently transmit with all flows in $G_{j}$, the scheduler checks whether the allocated slots for $G_{j}$ is sufficient for $f_{i}, s_{G_{j}} \geq s_{i}$. If yes, $f_{i}$ can be scheduled in $G_{j}$. Otherwise, the scheduler needs to check if the available number of slots is larger than the extra required slots of $f_{i}$, and schedules $f_{i}$ in $G_{j}$ if and only if $S>s_{i}-s_{G_{j}}$. If the condition is satisfied, the scheduler updates the allocated slots for all flows in $G_{j}, s_{G_{j}}=s_{i}$, and the available slots $S \leftarrow S-\left(s_{i}-s_{G_{j}}\right)$. If $f_{i}$ cannot concurrently transmit with some flows in the existing groups, the scheduler will schedule $f_{i}$ in a new group if the network resources is sufficient, and updates the group and available slots information accordingly. $f_{i}$ will be rejected if $f_{i}$ can not be accommodated in any existing groups and the available number of slots is not sufficient to support $f_{i}$.

\section{Simulation Results}

We use Network Simulator (NS2-2.32) and a real video source, "From Mars to China" in HDTV format (1920 x 1080i) [9] to validate the analysis. The simulated network is setup in a $10 \mathrm{~m} \times 10 \mathrm{~m}$ room. The senders and receivers of video flows are randomly distributed in the area. The simulation parameters are listed in TABLE 1. Signal bandwidth is $500 \mathrm{MHz}$. Senders use the maximal transmission power $P_{t}=0.1 \mathrm{~mW}$ and the background noise power is $-117 \mathrm{dBm} / \mathrm{MHz}$. mmWave signals fade fast in the air due to oxygen absorption and atmospheric attenuation and the path loss is measured as $71.5 \mathrm{~dB}$ at the reference distance $1.5 \mathrm{~m}$ with path loss exponent $\alpha=2$ [10]. The radiation angle of transceiver is $\theta=90$ degrees, and antenna gains are $G_{t}=G_{r}=360 / 90=4$. To further protect the concurrent transmissions of video flows and allow a certain level of angle estimation errors, we use a 15-degree angle margin in each side. The parameter $\eta$ is set as 0.293 that the achievable data rate at the reference distance is $1 \mathrm{Gbps}$. The duration of a superframe is $20 \mathrm{~ms}$, and the duration of a time slot is $17.3 \mu \mathrm{s}$. There are totally 1,000 time slots per super- 


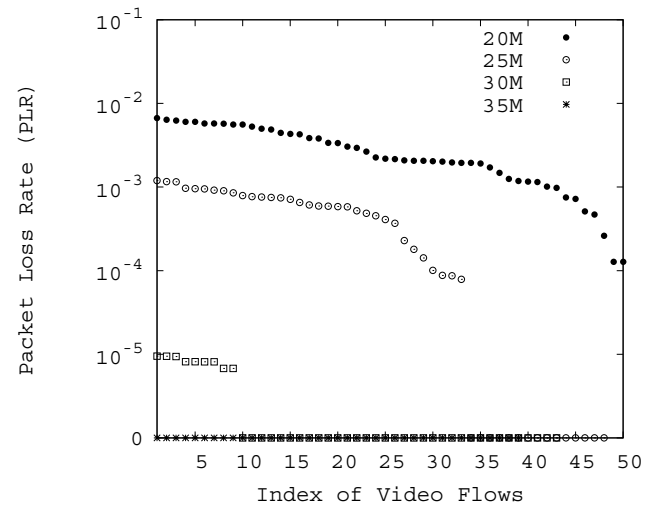

Figure 4. Packet Loss Rate (50 Flows, $n=10$ )

frame available for CTA, and the remaining $2.7 \mathrm{~ms}$ is used for beacon period and contention access period. Each simulation lasts $1200 \mathrm{~s}$. To eliminate the warming-up effects, the results of the initial 20s are not counted. We repeat the simulation 100 times with different random seeds to calculate the average value.

We first study the relationship of the reserved effective bandwidth and the buffer size. For delay sensitive multimedia flows, the maximal buffer size is determined according to the maximal tolerable queueing delay. With the delay bound of $60 \mathrm{~ms}$ and effective bandwidth $C=20 \mathrm{Mbps}$, the maximal buffer size is obtained as $B=60 * 20 / 8=$ 150 packets with payload 1000 bytes. The packet loss rate, which is the maximal number of dropped packet over the total number of transmitted packets, is tabulated in Table 2. For a higher reserved effective bandwidth, a larger buffer size can be applied for a given delay bounds, and a lower the packet loss performance is achieved. Both the simulation and analytical results show that, for $30 \mathrm{Mbps}$ effective bandwidth, the PLR is on the order of $10^{-6}$. Thus, the simulation validates the derived effective bandwidth for IPTV sources. We also observe that the average PLR performance does not change much with the aggregation parameter $n$. This is because our proposed concurrent scheduling scheme takes the transmission overheads into consideration and ensures the achieved throughput and PLR performance of admitted video flows.

We further study the PLR performance of each flow in Fig. 4. It is shown that the PLRs of most video flows are on the order of $10^{-3}, 10^{-4}$ and $10^{-6}$ for $20 \mathrm{Mbps}, 25 \mathrm{Mbps}$, 30 Mbps reserved bandwidth, respectively. Since many flows are concurrently transmitting, some concurrent flows may take advantages of the extra slots requested by other flows in the group and achieve better delay and throughput performance. With the proposed admission control and concurrent scheduling scheme, the delay and PLR performance of any single flow can always be guaranteed.

The number of video flows that can be accommodated

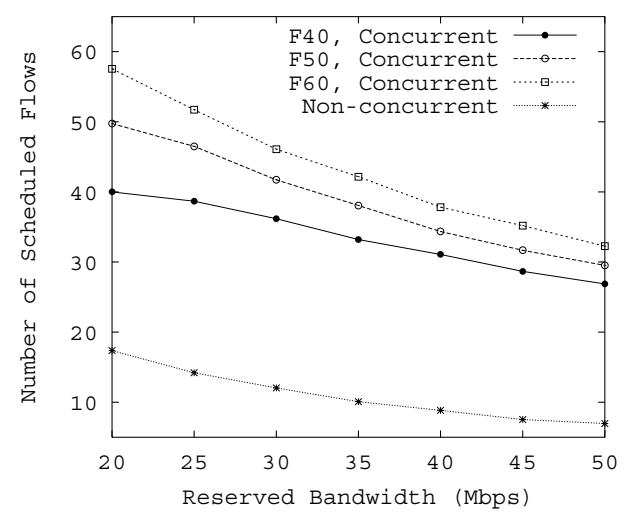

\section{Figure 5. Number of Admitted Flows vs Per- flow Reserved Bandwidth}

in the network is shown in Fig. 5. The maximal number of aggregated subframes is 10 , i.e., up to 10 subframes can be aggregated in one single transmission. When there are 40 video flows requesting bandwidth, only around 17 video flows can be accommodated with 20 Mbps reserved effective bandwidth using serial one-by-one transmission. The number of admitted video flows further decreases when a higher effective bandwidth is reserved for achieving better PLR performance. It is observed that only 10 flows can be supported with $35 \mathrm{Mbps}$ bandwidth reservation. Using the proposed concurrent scheduling scheme, all 40 flows can be accommodated in the network with $20 \mathrm{Mbps}$ bandwidth reservation, and 33 flows for $35 \mathrm{Mbps}$ reservation with a PLR below $10^{-6}$. With radiation angle $\theta=90$ degrees, the probability that two flows cannot transmit concurrently is as low as $1 / 16$. Thus, a new flow is very likely to be scheduled for concurrent transmission with existing flows. When more active flows join the network, the number of admitted video flows increases accordingly. With effective admission control, the packet loss performance can always be guaranteed and the network throughput is proportional to the number of scheduled video flows. By exploiting the high spatial multiplexing gain of mmWave WPANs, the proposed admission control and concurrent scheduling scheme can significantly improve the network throughput.

The maximal number of scheduled video flows under different aggregation parameter $n$ is investigated, and the results are shown in Fig. 6. Frame aggregation is generally used to improve the transmission efficiency and achieve high throughput. It can be seen that the maximal number of video flows that can be accommodated in the network increases from 34 for $n=5$ to 40 for $n=20$, with $35 \mathrm{Mbps}$ reserved bandwidth per flow. Less transmission overhead is involved when more subframes are aggregated in a single transmission. For bursty HD video flows, some video frames may exceed 300, 000 byte, which may be segmented 
Table 2. Packet Loss Rate (PLR)

\begin{tabular}{c||c|c|c|c|c}
\hline \hline \multicolumn{1}{c||}{ BW } & Buffer & \multicolumn{4}{c}{ Packet loss rate } \\
\hline (Mbps) & (Packets) & analysis & simulation, $\mathrm{n}=5$ & simulation, $\mathrm{n}=10$ & simulation, $\mathrm{n}=20$ \\
\hline 20 & 150 & $7.87 \mathrm{E}-3$ & $3.21 \mathrm{E}-3$ & $3.02 \mathrm{E}-3$ & $2.8 \mathrm{E}-3$ \\
\hline 25 & 187.5 & $6.35 \mathrm{E}-4$ & $4.12 \mathrm{E}-4$ & $3.66 \mathrm{E}-4$ & $3.34 \mathrm{E}-4$ \\
\hline 30 & 225 & $1.19 \mathrm{E}-6$ & $1.46 \mathrm{E}-6$ & $1.53 \mathrm{E}-6$ & $1.50 \mathrm{E}-6$ \\
\hline 35 & 262.5 & $5.79 \mathrm{E}-126$ & 0 & 0 & 0 \\
\hline$\geq 40$ & 300 & 0 & 0 & 0 & 0 \\
\hline \hline
\end{tabular}

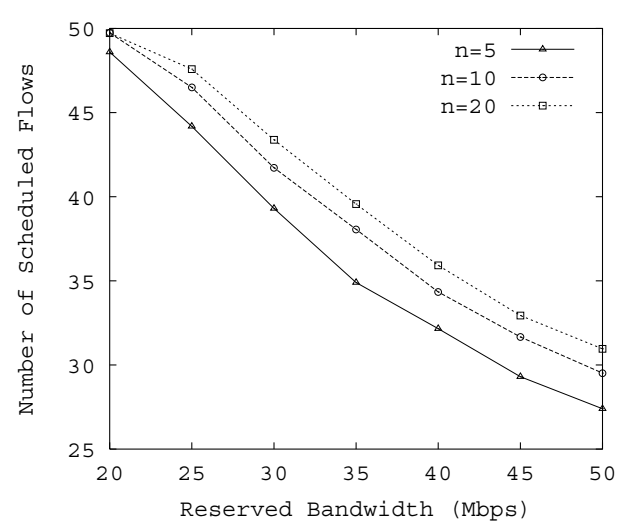

Figure 6. Number of Admitted Flows vs Aggregation Parameter

into more than 300 UDP packets of 1000 byte each. In this case, aggregating multiple packets (subframes) in one frame transmission can significantly improve the transmission efficiency and the number of admitted video flows.

\section{Conclusions}

In this paper, we have quantified the effective bandwidth of IPTV video sources using a simple two-level Markov traffic model. The minimal channel time allocated for each IPTV flow has been derived, considering the overheads of the protocol stack in mmWave WPANs. We have further proposed an admission control scheme and scheduling algorithm which take advantage of concurrent transmissions of mmWave-based WPANs with directional antenna. Extensive simulations with NS-2 using real video traces have validated our analysis and demonstrate the efficiency and effectiveness of the proposed schemes. Future research is needed to analytically study the performance of the proposed schemes in the presence of fast fading and shadowing in the mmWave channel.

\section{References}

[1] IEEE 802.15.3c TG. IEEE P802.15 high rate wireless personal area networks study group functional re- quirements standards development criteria, Nov. 2004.

[2] L. X. Cai, L. Cai, X. Shen, and J. W. Mark. Efficient resource management for mmwave wpans. In Proc. IEEE WCNC'07, Mar. 2007.

[3] J. Xu, X. Shen, J. W. Mark, and J. Cai. Adaptive transmission of multi-layered video over wireless fading channels. IEEE Trans. Wireless Commun., 6(6):2305$2314,2007$.

[4] IEEE 802.15.3 TG. IEEE std 802.15.3 $3^{T M}$ - 2003: Wireless medium access control (MAC) and physical layer (PHY) specifications for high rate wireless personal area networks (WPANs), Sept. 2003.

[5] F. Wan, L. Cai, and A. Gulliver. A simple, twolevel Markovian traffic model for IPTV video sources. Technical report, Department of Electrical \& Computer Engineering, University of Victoria, Mar. 2008.

[6] DSL Forum architecture \& transport working group. Triple-play services Quality of Experience (QoE) requirements. Technical Report TR-126, DSL Forum, Dec. 2006.

[7] B. Maglaris, D. Anastassiou, P. Sen, G. Karlsson, and J. Robbins. Performance models of statistical multiplexing in packet video communications. IEEE Trans. Commun., 36(7):834-844, July 1988.

[8] H. Harada et. al. Merged proposal: New PHY layer and enhancement of MAC for mmWave system proposal, Nov. 2007. http://www. ieee802.org/15/pub/TG3C_ CFPdoc\&Proposals.html\#Proposals.

[9] P. Seeling and M. Reisslein. Evaluating multimedia networking mechanisms using video traces. IEEE Potentials, 24(4):21-25, Oct./Nov. 2005.

[10] N. Moraitis and P. Constantinou. Indoor channel measurements and characterization at $60 \mathrm{GHz}$ for wireless local area network applications. IEEE Trans. Antennas Propag., 53:3180-3189, Dec. 2004. 